\title{
Effects of surface characteristics of activated carbon on the adsorption of 2-methylisobornel (MIB) and geosmin from natural water
}

\author{
Jianwei Yu ${ }^{\mathrm{a}}$, Min Yang ${ }^{\mathrm{a}, *}$, Tsair-Fuh Lin ${ }^{\mathrm{b}}$, Zhaohai Guo ${ }^{\mathrm{a}}$, \\ Yu Zhang ${ }^{a}$, Junnong $\mathrm{Gu}^{\mathrm{c}}$, Suxia Zhang ${ }^{\mathrm{c}}$ \\ a SKLEAC, Research Center for Eco-Environmental Sciences, Chinese Academy of Sciences, 18 Shuangqing Road, \\ P.O. Box 2871, Beijing 100085, China \\ ${ }^{\mathrm{b}}$ Department of Environmental Engineering, National Cheng Kung University, Tainan City 70101, Taiwan \\ ${ }^{\mathrm{c}}$ Beijing Waterworks (Group) Co., Ltd., Beijing 100085, China
}

Received 19 October 2006; received in revised form 22 January 2007; accepted 22 January 2007

\begin{abstract}
Five powdered activated carbons (PACs), including one fruit-based, one wood-based, and three bituminous coal-based, were selected for the investigation of the effects of surface characteristics of activated carbon on the adsorption of 2-methylisobornel (MIB) and geosmin. Characterization of the carbons was performed using nitrogen adsorption, Fourier transform infra-red (FTIR) spectroscopy and X-ray photoelectron spectroscopy (XPS). All the carbons showed a broad absorption band in the $1300-1000 \mathrm{~cm}^{-1}$ region, which can be assigned to C-O stretching and O- $\mathrm{H}$ bending modes of alcoholic, phenolic, and carboxylic groups in FTIR spectra. The contents of $\mathrm{O}$ and $\mathrm{C}$ on the surfaces of carbons were acquired from the data of XPS analysis. Statistical analyses on the relationship between the adsorption capacities and different carbon surface parameters (O content, $\mathrm{C}=\mathrm{O}$ and $\mathrm{C}-\mathrm{O}$ chemical group contents, the surface area, different pore volumes, iodine number and methylene blue number) were performed using Spearman rank correlation method. A good linear relationship between the adsorption capacities for MIB and geosmin and the micropore volumes was acquired. Both of iodine number and methylene blue number, the two most often used parameters for the evaluation of activated carbon quality, and other parameters, such as meso and total pore volumes, surface area, $\mathrm{O}$ and $\mathrm{C}-\mathrm{O}$ contents were found to be insignificant in correlation with the adsorption capacities of MIB and geosmin. The volume of micropores could be used as an effective indicator for the selection of PAC for the removal of both MIB and geosmin.
\end{abstract}

(c) 2007 Elsevier B.V. All rights reserved.

Keywords: Activated carbon characteristic; Adsorption; Geosmin; 2-Methylisobornel; Odor removal

\section{Introduction}

2-Methylisobornel (MIB) and geosmin are two important earthy and musty-smelling compounds that are produced as a secondary metabolite by some microorganisms in natural water. These compounds can be perceived by most consumers as musty-earthy odors even at levels as low as $10 \mathrm{ng} / \mathrm{L}[1,2]$, requiring a high efficient treatment to achieve an extremely low concentration in the finished water. Powdered activated carbon (PAC) adsorption is an effective and most often used mean for controlling MIB and geosmin related odors in drinking water, because of its relatively low cost and flexibility [1,3-5]. However, previous studies indicate that the adsorption

\footnotetext{
* Corresponding author. Tel.: +86 1062923475 ; fax: +86 1062923541 . E-mail address: yangmin@ rcees.ac.cn (M. Yang).
}

efficiency of such contaminants is very dependent on the types of PAC $[1,6-8]$. Some researchers have found that the ligniteand wood-based chemically activated carbons displayed inferior adsorption for MIB compared with bituminous-based carbons $[9,10]$. Unfortunately, the basis for the PAC selection has been empirical, and the carbon quality is typically evaluated by using iodine number, methylene blue number and specific surface area as the main criteria $[11,12]$. However, some researchers found that these parameters could not represent the carbon adsorption performance in the removal of MIB and geosmin [9].

The efficiency of a carbon for removing a given pollutant depends largely on its characteristics, including surface chemistry (surface functional groups) and pore structure (surface area, pore volume, pore size distribution, etc.) [13,14]. Surface carbon-oxygen groups are the most common oxygenbearing functional groups found on activated carbon surfaces [15]. Kaneko et al. [16] showed that the removal of the acidic 
functional groups from activated carbon enhanced the adsorption of both relatively polar and nonpolar organic compounds from aqueous solution. By investigating the correlation between MIB adsorption capacity and the oxygen content of carbon, Considine et al. [17] also reported that the increase of carbon-oxygen content at a constant pore volume leaded to a decrease in the amount of MIB adsorbed. On the other hand, a carbon containing abundant pores in the size range of the target molecule is expected to have a high adsorption potential. Some researchers have found that the presence of micropores $(<20 \AA)$ is important for the removal of MIB and geosmin, which have similar spherical diameters of about $6 \AA$. Nowack et al. [18] suggested that MIB adsorption mainly occurred in the pore size range of 5.5-63 $\AA$. Newcombe et al. [19] found that MIB adsorption was mainly related to the micropore volumes within the pore size range between 10 and $12 \AA$. However, the studies on the factors affecting the adsorption of MIB and geosmin by activated carbon are insufficient, and the selection of activated carbon has still been relied on the conventional parameters like iodine number and methylene blue number.

The purpose of this study is to establish an effective method for selecting activated carbon for the adsorption of MIB and geosmin by clarifying the factors affecting carbon adsorption performance. Five commercial activated carbons (including one fruit-based, one wood-based, and three bituminous coal-based) were selected, and were characterized using nitrogen adsorption, Fourier transform infra-red (FTIR) spectroscopy, and X-ray photoelectron spectroscopy (XPS). By using a statistical analysis method, the different properties of the carbons were related to the adsorption capacities of MIB and geosmin and the main rational for the selection of PAC to remove MIB and geosmin was established.

\section{Experimental}

\subsection{Materials}

MIB and geosmin were purchased from Sigma-Aldrich Co., USA, at a concentration of 10 and $2 \mathrm{mg} / \mathrm{mL}$ in methanol, respectively. Stock solutions of $1 \mathrm{mg} / \mathrm{L}$ were prepared by diluting the methanol solution with ultra-pure water (resistivity $\geq 18 \mathrm{~m} \Omega \mathrm{cm}$ ). The natural water used in this study was collected from Miyun Reservoir, Beijing, China. Water samples were stored at $4{ }^{\circ} \mathrm{C}$ in the dark to limit biological activity, and characteristics of the natural water are shown in Table 1.

Five commercial powdered activated carbons (PACs) were used in this study. Three bituminous coal-based carbons B1 (Tangshan Huaneng Carbon Corporation, China), B2 and B3 (Ningxia Taixi Carbon Corporation, China), a fruit shell-based

Table 1

Characteristics of miyun reservoir water

\begin{tabular}{lc}
\hline $\mathrm{pH}$ & 8.10 \\
Alkalinity, $\mathrm{mg} / \mathrm{L} \mathrm{CaCO}_{3}$ & 142 \\
Hardness, $\mathrm{mg} / \mathrm{L} \mathrm{CaCO}$ & 182 \\
Total organic carbon, $\mathrm{mg} / \mathrm{L}$ & 2.69 \\
Turbidity, NTU & 1.32 \\
\hline
\end{tabular}

carbon F (Tangshan Huaneng Carbon Corporation, China), and a wood-based carbon W (Shanxi Xinhua Carbon Corporation, China) were selected for the study. Prior to experiments, the PAC samples were washed using ultra-pure water and dried overnight at $110{ }^{\circ} \mathrm{C}$ to remove excess water, and then cooled and stored in a desiccator.

\subsection{Characterization of activated carbons}

\subsubsection{Surface area and pore size distribution}

The surface area and pore size distribution of the PAC samples were determined by nitrogen adsorption/desorption isotherms measured at $77 \mathrm{~K}$ using an AUTOSORB (Quantachrome, USA) computer-controlled surface analyzer. All the samples were initially outgassed for $12 \mathrm{~h}$ at $473 \mathrm{~K}$ in vacuum. The specific surface area $\left(S_{\mathrm{BET}}\right)$ was determined according to the BET model. The density functional theory (DFT) was used to calculate micropore volumes $(<20 \AA)$, mesopore volumes $(20-500 \AA)$ and pore size distribution from the nitrogen desorption isotherms with the software supplied by Quantachrome, USA.

\subsubsection{Fourier transform infra-red (FTIR) spectroscopy}

The FTIR spectra of the carbon samples were characterized on a NEXUS 670 FTIR spectrophotometer (Nicolet, USA). The carbon samples were mixed with $\mathrm{KBr}$ at a ratio of $1: 300$ to form $\mathrm{KBr}$ pellets. The pellets were then dried overnight at $90^{\circ} \mathrm{C}$ before the spectra were determined. The pellet samples were recorded for their FTIR spectra from 4000 to $500 \mathrm{~cm}^{-1}$ at a resolution of $4 \mathrm{~cm}^{-1}$ and with 200 scans for each sample. Before each measurement, the instrument was run to collect the background spectrum of air, which was then automatically subtracted from the sample spectrum [20,21].

\subsubsection{X-ray photoelectron spectroscopy (XPS)}

XPS spectra were obtained with an EscaLab220i-XL (V.G. Scientific Ltd., UK) photoelectron spectrometer using a nonmonochromatised $\mathrm{Al} \mathrm{K} \alpha$ radiation (energy $1486.6 \mathrm{eV}$ ). The $\mathrm{X}$-ray power source was operated at $300 \mathrm{~W}$. The measurements were performed under near vacuum condition, with a pressure lower than $3 \times 10^{-7} \mathrm{~Pa}$. The survey scans were collected from 0 to $1200 \mathrm{eV}$. The high-resolution scans were performed over the 280-296 eV and 526-542 eV (C 1s and O 1s spectra, respectively) for the tested samples. For calibration purposes, the C 1s electron bond energy corresponding to graphitic carbon was referenced to $284.8 \mathrm{eV}$. After subtraction of a shirley background, the curve fitting was performed using the non-linear least-squares algorithm assuming a mixed Gaussian/Lorentzian peak shape (the ratio of Gaussian to Lorentzian form was 0.3). This peak-fitting procedure was repeated until an acceptable fit was obtained. The positions of the deconvoluted peaks (binding energy-BE) were determined from both literature data [21-24] and empirically derived values.

\subsection{Equilibrium adsorption test}

The bottle-point technique was used to conduct the adsorption isotherm tests for MIB and geosmin on PAC [25]. A 

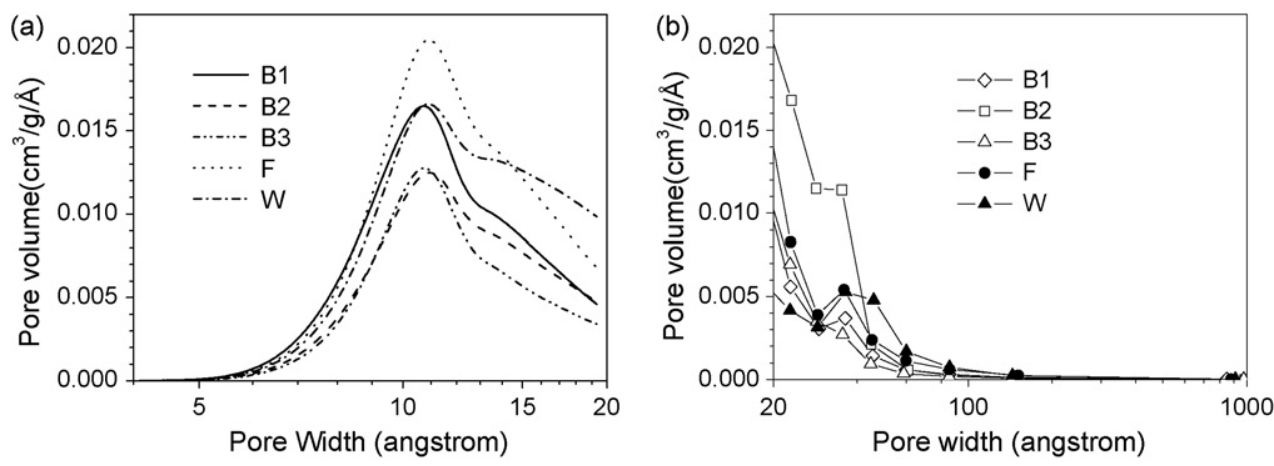

Fig. 1. Pore size distributions of the PAC samples: (a) micropore size distribution; (b) mesopore size distribution.

constant initial concentration of MIB and geosmin at $100 \mathrm{ng} / \mathrm{L}$ was used by spiking the stock solution to natural water, which is a concentration commonly found in the influent for waterworks [26-28]. The PAC was added in a slurry form of $10 \mathrm{mg} / \mathrm{mL}$, which was prepared by mixing $10 \mathrm{~g}$ of the oven-dried PAC in $1 \mathrm{~L}$ of ultra-pure water. The carbon doses were varied between 2 and $30 \mathrm{mg} / \mathrm{L}$. The bottles were sealed and agitated on a rotary mixer for 3-5 days to reach adsorption equilibrium [4]. After this, the solution was filtered through a glass fiber filter (Whatman GF/C, UK). The final concentration of MIB and geosmin was determined immediately by using headspace solid phase microextraction (SPME) combined with gas chromatography/mass spectrometry (GC/MS). The detection limits were $1 \mathrm{ng} / \mathrm{L}$ for geosmin and $3 \mathrm{ng} / \mathrm{L}$ for $\mathrm{MIB}$, respectively $[29,30]$.

\section{Results and discussion}

\subsection{Physical characterization of the activated carbon}

Fig. 1 gives the pore size distributions of the carbons. All the carbon samples exhibit similar micropore size distribution, and micropores of the carbons are concentrated in the 8-20 width range. All the carbons exhibit the largest volumes at a pore width of about $11 \AA$, which is a favorite adsorption size for MIB according to Newcombe et al. [19]. As for the mesopore, all of the carbons except B2 indicate a similar size distribution. The data of specific surface area $\left(S_{\mathrm{BET}}\right)$, total pore volume $\left(V_{\mathrm{tot}}\right)$, micropore volume $\left(V_{\text {micro }}\right)$, mesopore volume $\left(V_{\text {meso }}\right)$ as well as the usually used carbon capacity indicators of iodine number and methylene blue number are summarized in Table 2. It can be seen that all the carbons are essentially microporous. For the five carbon samples, the ranking of surface area is in accordance with total pore volume as $\mathrm{B} 3<\mathrm{B} 1<\mathrm{W}<\mathrm{B} 2<\mathrm{F}$. In spite of its high mesopore volume, B2 shows a relatively small surface area compared with carbon $\mathrm{F}$ because of its small micropore volume. The fruit shell-based carbon $\mathrm{F}$ and wood-based carbon $\mathrm{W}$ exhibit larger micropore volumes than other three bituminous coal-based carbons (B1-B3). These differences may be ascribed to the primary raw material nature [31], or the difference of manufacturing processes.

\subsection{Chemical characterization of the activated carbon}

The FTIR spectra for the carbon samples are shown in Fig. 2. Below $2000 \mathrm{~cm}^{-1}$, the FTIR spectra of the carbons

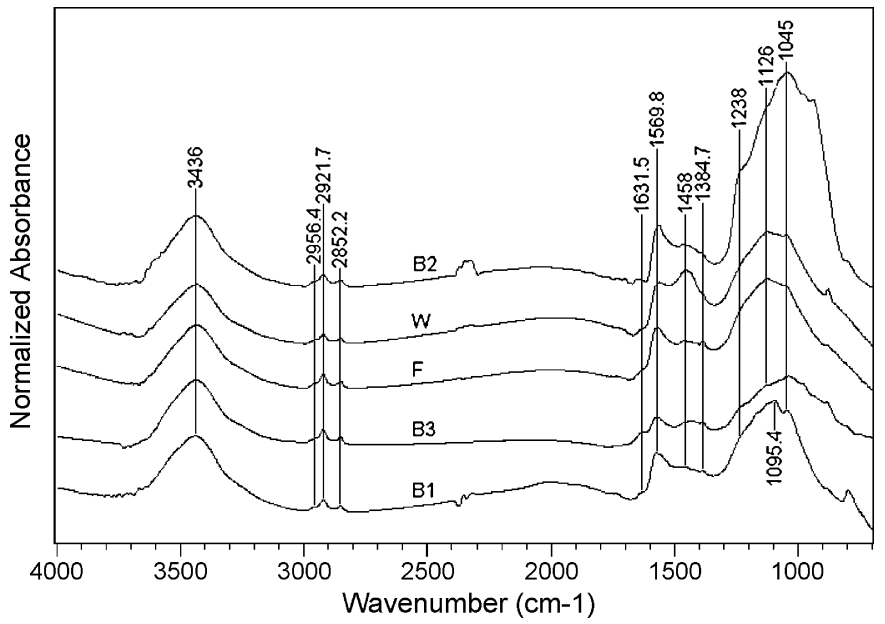

Fig. 2. FTIR spectra of the PAC samples.

Table 2

Characteristics of the PAC samples

\begin{tabular}{lcllccc}
\hline Samples & $S_{\text {BET }}\left(\mathrm{m}^{2} / \mathrm{g}\right)$ & $V_{\text {tot }}\left(\mathrm{cm}^{3} / \mathrm{g}\right)$ & $V_{\text {micro }}\left(\mathrm{cm}^{3} / \mathrm{g}\right)$ & $V_{\text {meso }}\left(\mathrm{cm}^{3} / \mathrm{g}\right)$ & $\begin{array}{l}\text { Iodine number } \\
(\mathrm{mg} / \mathrm{g})^{\mathrm{a}}\end{array}$ \\
\hline B1 & 805 & 0.49 & 0.32 & 0.17 & 926 & 174 \\
B2 & 943 & 0.60 & 0.28 & 0.32 & 1026 & 192 \\
B3 & 661 & 0.39 & 0.25 & 0.14 & 955 & 117 \\
F & 1158 & 0.71 & 0.45 & 0.26 & 962 & 186 \\
W & 828 & 0.57 & 0.33 & 0.24 & 188 \\
\hline
\end{tabular}

\footnotetext{
${ }^{a}$ Data from carbon corporations.
} 
Table 3

Distribution of oxygen-bearing structures (At.\%) from O 1s XPS spectra and the atomic ratios of O and C on PAC surfaces

\begin{tabular}{llllr}
\hline Samples & \multicolumn{2}{l}{ Groups from O 1s fitting (At.\%) } & O (At.\%) & C (At.\%) \\
\cline { 2 - 4 } & $\mathrm{C}=\mathrm{O}(532.2 \pm 0.1)^{\mathrm{a}}$ & $\mathrm{C}-\mathrm{O}(533.7 \pm 0.2)^{\mathrm{a}}$ & $\mathrm{H}_{2} \mathrm{O}$ adsorbed $(536.0 \pm 0.3)^{\mathrm{a}}$ & 7.38 \\
\hline B1 & 3.42 & 2.75 & 1.21 & 92.62 \\
B2 & 2.95 & 4.81 & 3.94 & 88.30 \\
B3 & 3.10 & 3.83 & 1.27 & 91.81 \\
F & 3.63 & 2.05 & 0.81 & 9.19 \\
W & 4.86 & 2.29 & 1.78 & 9.49 \\
\hline
\end{tabular}

${ }^{\text {a }}$ Binding energy (eV).

display typical absorption of surface functional groups and structural oxygen species. There is a broad absorption band in the $1300-1000 \mathrm{~cm}^{-1}$ region for all the carbon samples, which can be assigned to $\mathrm{C}-\mathrm{O}$ stretching and $\mathrm{O}-\mathrm{H}$ bending modes of alcoholic, phenolic, and carboxylic groups [32,33]. In this region, carbon B2 demonstrates significantly higher band intensity, suggesting the existence of abundant $\mathrm{C}-\mathrm{O}$ and $\mathrm{O}-\mathrm{H}$ structures. The presence of a relatively strong band at $1569 \mathrm{~cm}^{-1}$ can be attributed to conjugated systems such as diketone, keto-ester and keto-enol structures [23]. Compared with other four carbon samples, carbon $\mathrm{W}$ displays strong absorbance in the region $1470-1380 \mathrm{~cm}^{-1}$. This region includes a series of overlapping absorption bands ascribable to the deformation vibration of surface hydroxyl groups and in-plane vibrations of $\mathrm{C}-\mathrm{H}$ in various $\mathrm{C}=\mathrm{C}-\mathrm{H}$ structures [34]. The bands observed at 2921.7, 2956.4 and $2852.2 \mathrm{~cm}^{-1}$ can be ascribed to asymmetric and symmetric $\mathrm{C}-\mathrm{H}$ stretching vibrations in $-\mathrm{CH},-\mathrm{CH}_{2}$ and $-\mathrm{CH}_{3}$ groups $[21,32]$, suggesting the existence of some aliphatic species on the carbons. On the other hand, in all the recorded spectra, an obvious band of O-H stretching vibration $\left(3600-3200 \mathrm{~cm}^{-1}\right.$ ) $[22,35]$, due to surface hydroxylic groups and chemisorbed water, was observed.

The XPS scan spectra of the five carbons indicate the presence of two distinct peaks attributed to carbon and oxygen, respectively. The high-resolution $\mathrm{C}$ 1s and $\mathrm{O}$ 1s spectra (Fig. 3) show the presence of several peaks for each element. Deconvolution of the $\mathrm{C} 1 \mathrm{~s}$ spectra yields several peaks with different binding energies (BE) representing graphitic carbon $(284.7 \mathrm{eV})$ as the dominating species on the surface, carbon present in phenolic, alcohol or ether groups $(286.2 \mathrm{eV})$, carbonyl or quinone groups $(287.4 \mathrm{eV})$, carboxyl or ester groups $(288.7 \mathrm{eV})$, and a satellite signal due to $\pi-\pi^{*}$ transitions in aromatic ring $(290.2 \mathrm{eV})$. These assignments agree well with the extensive studies made by other researchers $[21,23,24]$. The $\mathrm{O} 1$ s spectra for the carbon samples display three main peaks corresponding to the $\mathrm{C}=\mathrm{O}$ groups $(532.2 \mathrm{eV}), \mathrm{C}-\mathrm{O}$ groups $(533.7 \mathrm{eV})$ and adsorbed water molecules $(536 \mathrm{eV})[21,22]$.

The deconvolution results of $\mathrm{O} 1 \mathrm{~s}$ spectra as well as the content of oxygen and carbon composition of PAC samples derived from XPS are presented in Table 3 . The analysis of $\mathrm{O} 1 \mathrm{~s}$ envelope shows the existence of varieties of the $\mathrm{C}=\mathrm{O}$ and $\mathrm{C}-\mathrm{O}$ groups, and among them, phenols are the main compounds on carbon $\mathrm{B} 2$ and B3, which is in agreement with the FTIR results. This difference may be due to the different activation processes of carbons [36].

\subsection{Adsorption studies}

Fig. 4 shows the adsorption isotherms of MIB and geosmin on the five PACs in natural water fitted with Freundlich model. It is clear that, for both MIB and geosmin, different carbons demonstrate quite different adsorption capacities. Carbon F exhibits the highest adsorption capacities for both MIB and geosmin while carbon B3 exhibits the lowest ones. At an equilibrium concentration of $10 \mathrm{ng} / \mathrm{L}$, the adsorption capacities of MIB and geosmin on carbon F were almost 4.5 and 3.5 times those on carbon B3, respectively. For the parameters of BET surface area, total pore volume, iodine number and methylene blue number, although carbon B2 all has markedly higher values than $\mathrm{W}$, its adsorption capacities for both MIB and geosmin are far lower than those of $\mathrm{W}$, indicating that these indicators are not suitable parameters for evaluating the performance of activated carbons for the removals of MIB and geosmin from natural water. Among the five carbons selected, the bituminous-based carbons (carbon B1-B3) display inferior adsorption compared with fruit shell-based (carbon F) and wood-based (carbon W) carbon for adsorption of MIB and geosmin, which is contrary to the results of some other references $[9,10]$. So the origins of raw materials should not be a decisive factor in the adsorption of MIB and geosmin by carbons.

To find the decisive factors affecting the adsorption of MIB and geosmin, statistical analyses on the relationship between the adsorption capacities and different carbon surface parameters ( $\mathrm{O}$ content, $\mathrm{C}=\mathrm{O}$ and $\mathrm{C}-\mathrm{O}$ chemical group contents, the surface area, different pore volumes, iodine number and methylene blue number) were performed with SPSS 11.0 based on Spearman rank correlation method. The Spearman rank coefficients $(r, P)$ were calculated, and a $P$-value less than 0.05 is considered statistically significant for all analyses. The correlation analysis results are summarized in Table 4. According to the Spearman rank, the adsorption capacities for both MIB and geosmin were highly correlated with micropore volumes of activated carbons with $r=1.00, P=0.000$ for MIB and $r=0.90, P=0.037$ for geosmin, respectively. However, the two most often used parameters for the evaluation of activated carbon quality, iodine number and methylene blue number, were both insignificant in correlation with the adsorption capacities of MIB and geosmin. Although there was also a positive trend for meso and total pore volumes and surface area, and a negative trend for $\mathrm{O}$ and $\mathrm{C}-\mathrm{O}$ content with adsorption capacities, none of these were statistically significant. Furthermore, no significant correlation was found between the adsorption capacities of MIB and geosmin 
(a)
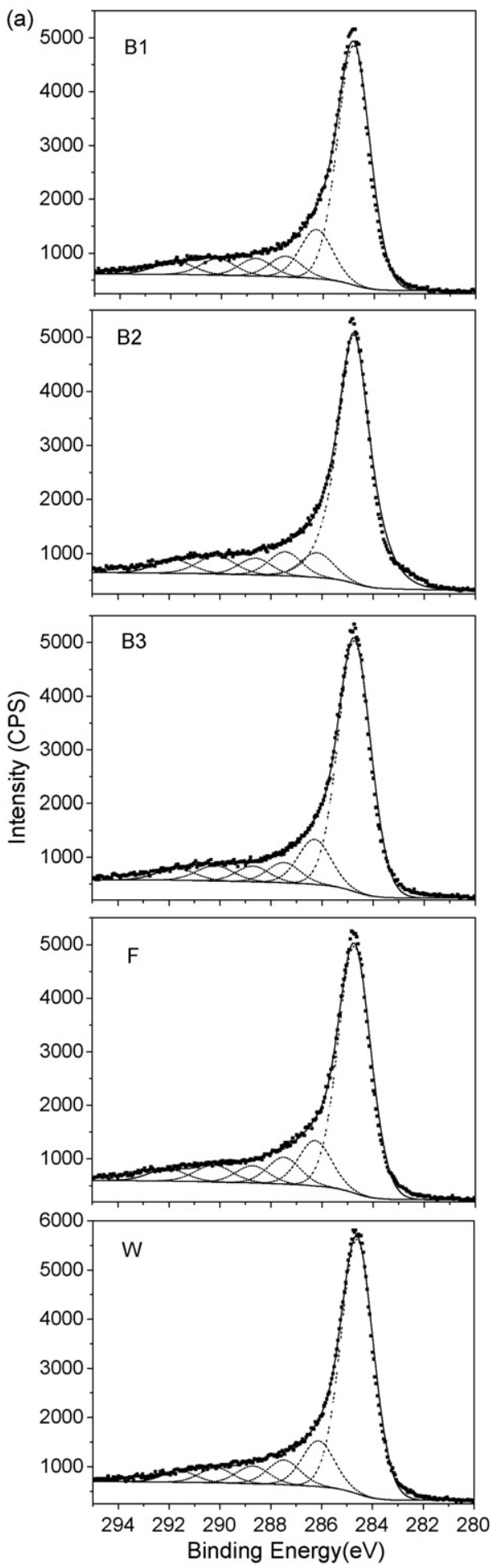

(b)
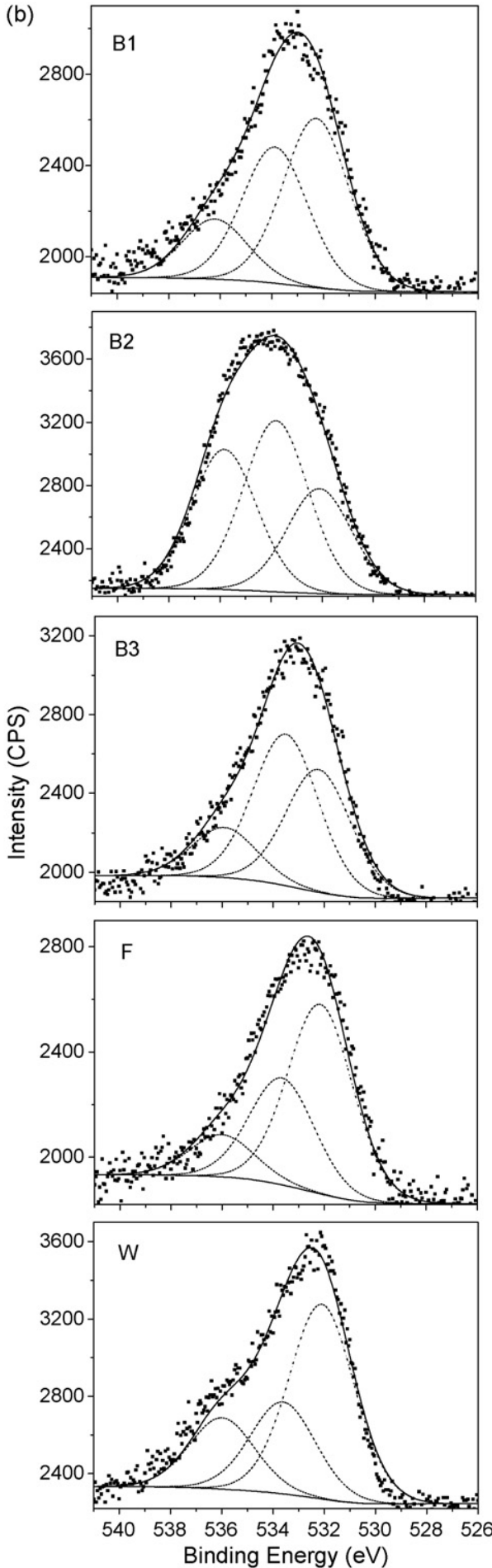

Fig. 3. $\mathrm{C}$ 1s and O 1s XPS spectra of the PAC samples: (a) C 1s; (b) O 1s.

and the micropore volumes of $10-12 \AA(r=0.80, P=0.104$ for MIB and $r=0.50, P=0.391$ for geosmin, respectively), which was different from the result that Newcombe et al. [19] acquired.

Fig. 5 presents the relationship between the micropore volumes and amount of MIB or geosmin adsorbed by the carbon at an equilibrium adsorbate concentration of $10 \mathrm{ng} / \mathrm{L}$. It is clear that the adsorption capacities increase almost linearly with the increase of micropore volumes of carbons, indicating that the micropore volume measured by nitrogen adsorption might be a good parameter for evaluating the adsorption capacities of carbons for both MIB and geosmin. MIB and geosmin are generally treated as hydrophobic compounds with molecular sizes of about $6 \AA$ [8]. The most likely adsorption mechanism is hydrophobic attraction to the carbon surface, and the compounds would be preferentially adsorbed into the micropores. On the other hand, in spite of similar micropore volumes $\left(0.33\right.$ and $0.32 \mathrm{~cm}^{3} / \mathrm{g}$ for W 

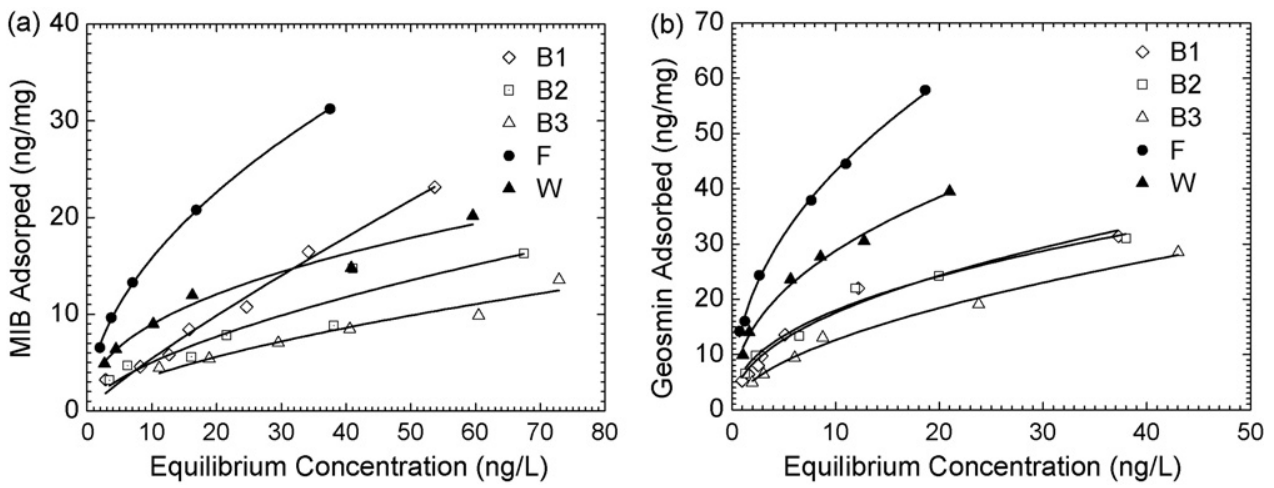

Fig. 4. Freundlich adsorption isotherms of PACs for MIB and geosmin in natural water: (a) MIB; (b) geosmin.

Table 4

Results of Spearman rank correlation between adsorption capacities of MIB and geosmin and carbon characteristics

\begin{tabular}{|c|c|c|c|c|}
\hline Carbon characteristics & \multicolumn{2}{|c|}{ MIB adsorbed ${ }^{\mathrm{a}}$ (ng/mg) } & \multicolumn{2}{|c|}{ Geosmin adsorbed $^{\mathrm{a}}(\mathrm{ng} / \mathrm{mg})$} \\
\hline $\mathrm{O}(\mathrm{At} . \%)$ & -0.500 & 0.391 & -0.200 & 0.747 \\
\hline $\mathrm{C}-\mathrm{O}(\%)$ & -0.800 & 0.104 & -0.700 & 0.188 \\
\hline$S_{\mathrm{BET}}\left(\mathrm{m}^{2} / \mathrm{g}\right)$ & 0.700 & 0.188 & 0.800 & 0.104 \\
\hline$V_{\text {tot }}(\mathrm{mL} / \mathrm{g})$ & 0.700 & 0.188 & 0.800 & 0.104 \\
\hline $\mathrm{V}_{\text {micro }(10-12 \AA)}{ }^{\mathrm{c}}(\mathrm{mL} / \mathrm{g})$ & 0.800 & 0.104 & 0.500 & 0.391 \\
\hline Iodine number ${ }^{\mathrm{d}}(\mathrm{mg} / \mathrm{g})$ & 0.300 & 0.624 & 0.600 & 0.285 \\
\hline Methylene blue number ${ }^{\mathrm{d}}(\mathrm{mg} / \mathrm{g})$ & 0.300 & 0.624 & 0.600 & 0.285 \\
\hline
\end{tabular}

\footnotetext{
${ }^{a}$ Mass MIB or geosmin adsorbed at an equilibrium concentration of $10 \mathrm{ng} / \mathrm{L}$.

b Total micrpore volumes.

c Micropore volumes between 10 and $12 \AA$.

d Data as shown in Table 2.
}

and $\mathrm{B} 1$, respectively), carbon $\mathrm{W}$ demonstrates higher adsorption capacities for MIB and geosmin than B1. The higher mesopore volume of $\mathrm{W}$ might be responsible for this difference. However, further studies are required to explain the reason.

Considine et al. [17] has shown that surface chemistry plays a role in MIB and geosmin adsorption, but the study was conducted in maintaining a constant pore volume distribution. In this study, the statistical results showed there was no significant correlation between the adsorption capacity and the oxygen content

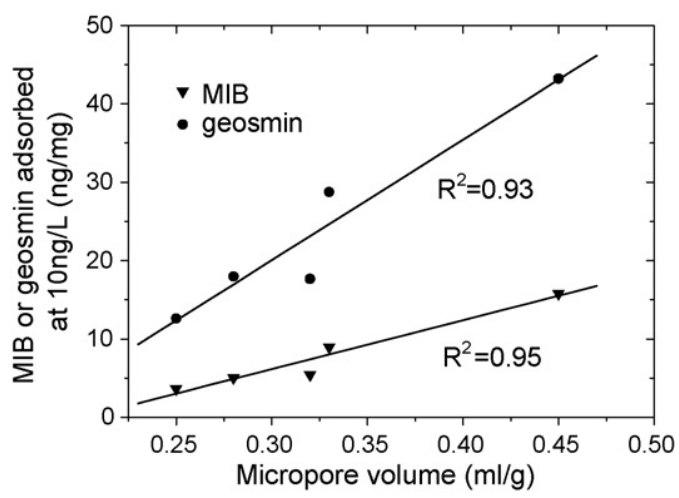

Fig. 5. Relationship between surface concentrations of MIB and geosmin and micropore volumes of activated carbons. or the functional groups. Surface chemistry might influence the adsorption capacities of MIB and geosmin to some extent. However, this influence could be ignored in drinking water treatment since the concentration of NOM is about 5-6 orders of magnitude larger than that of MIB and geosmin [10,19]. Similar with previous studies $[1,3,5]$, regardless of the pore structure or surface chemistry, geosmin is easier to adsorb on activated carbons than MIB. This may be due to the difference of structure between MIB and geosmin. Geosmin has a slightly lower solubility and molecular weight, and has a flatter structure, which may render it more amenable to adsorption in the slit-shaped pores of the activated carbon [3].

Although Newcombe et al. [19] found that MIB adsorption was mainly related to the micropore volumes within the pore size range between 10 and $12 \AA$, their results were not replicated in our study. It is possible that the pore size range between 10 and $12 \AA$ is too narrow to give a precise pore volume. So the micropore volume might be a better parameter to represent the adsorption capacity for MIB and geosmin.

\section{Conclusions}

In this study, five powdered activated carbons (PACs) were evaluated for MIB and geosmin removal from natural water and 
the main factors affecting the adsorption of MIB and geosmin were investigated. The fruit shell-based carbon shows the highest adsorption capacity than wood- and bituminous coal-based carbons, which is mainly attributed to its larger micropore volumes. By correlating the adsorption data with different carbon property parameters using Spearman rank correlation method, a high correlation was acquired between the adsorption capacities of MIB and geosmin and the micropore volumes of activated carbons. Both of iodine number and methylene blue number, two of the most often used parameters for the evaluation of activated carbon quality, were found to be insignificant in correlation with the adsorption capacities of MIB and geosmin. The relationship between the adsorption capacities of carbons for MIB and geosmin and other parameters, such as meso and total pore volumes, surface area, $\mathrm{O}$ and $\mathrm{C}-\mathrm{O}$ contents were also found to be statistically insignificant. The volume of micropores could be used as an effective indicator for the selection of PAC for the removal of both MIB and geosmin.

\section{Acknowledgments}

We acknowledge the support provided by Beijing Science Council of China (D0605004040421), National Natural Science Foundation of China (Contract No. 50678166) and the State High Tech Research and Development Project of China for younger researchers (2004AA649280).

\section{References}

[1] S. Lalezary, M. Pirbazari, M.J. McGuire, Evaluating activated carbons for removing low concentrations of taste and odour producing organics, J. Am. Water Works Assoc. 78 (1986) 76-82.

[2] I.H. Suffet, A. Corado, D. Chou, M.J. McGuire, S. Butterworth, AWWA taste and odor survey, J. Am. Water Works Assoc. 88 (1996) 168 180.

[3] D. Cook, G. Newcombe, P. Sztajnbok, The application of powdered activated carbon for MIB and geosmin removal: predicting PAC doses in four raw waters, Water Res. 35 (2001) 1325-1333.

[4] M.R. Graham, R.S. Summers, M.R. Simpson, B.W. MacLeod, Modeling equilibrium adsorption of 2-methylisoborneol and geosmin in natural waters, Water Res. 34 (2000) 2291-2300.

[5] S. Lalezary-Craig, M. Pirbazari, M.S. Dale, T.S. Tanaka, M.J. McGuire, Optimising the removal of geosmin and 2-methylisoborneol by powdered activated carbon, J. Am. Water Works Assoc. 80 (1988) 7380.

[6] C. Ng, J.N. Losso, W.E. Marshall, R.M. Rao, Freundlich adsorption isotherms of agricultural by-product-based powdered activated carbons in a geosmin-water system, Bioresource Technol. 85 (2002) 131135.

[7] G. Newcombe, J. Morrison, C. Hepplewhite, Simultaneous adsorption of MIB and NOM onto activated carbon. I. Characterization of the system and NOM adsorption, Carbon 40 (2002) 2135-2146.

[8] P. Pendleton, S.H. Wong, R. Schumann, G. Levay, R. Denoyel, J. Rouquero, Properties of activated carbon controlling 2-methylisoborneol adsorption, Carbon 35 (1997) 1141-1149.

[9] G. Chen, B.W. Dussert, I.H. Suffet, Evaluation of granular activated carbons for removal of methylisoborneol to below odor threshold concentration in drinking water, Water Res. 31 (1997) 1155-1163.

[10] G. Newcombe, M. Drikas, R. Hayes, Influence of characterized natural organic material on activated carbon adsorption. II. Effect on pore volume distribution and adsorption of 2-methylisoborneol, Water Res. 31 (1997) 1065-1073.
[11] L. Li, P.A. Quinlivan, D.R.U. Knappe, Effects of activated carbon surface chemistry and pore structure on the adsorption of organic contaminants from aqueous solution, Carbon 40 (2002) 2085-2100.

[12] I.N. Najm, V.L. Snoeyink, Y. Richard, Effect of initial concentration of a soc in natural-water on its adsorption by activated carbon, J. Am. Water Works Assoc. 83 (1991) 57-63.

[13] J. Lahaye, The chemistry of carbon surfaces, Fuel 77 (1998) 543-547.

[14] L.R. Radovic, I.F. Silva, J.I. Ume, J.A. Menendez, C.A.L.Y. Leon, A.W. Scaroni, An experimental and theoretical study of the adsorption of aromatics possessing electron-withdrawing and electron-donating functional groups by chemically modified activated carbons, Carbon 35 (1997) 1339-1348.

[15] R.C. Bansal, J.B. Donnet, F. Stoeckli, Active Carbon, Marcel Dekker Inc., New York, 1988, pp. 27-35.

[16] Y. Kaneko, M. Abe, K. Ogino, Adsorption characteristics of organic compounds dissolved in water on surface-improved activated carbon fibers, Colloid Surface 37 (1989) 211-222.

[17] R. Considine, R. Denoyel, P. Pendleton, R. Schumann, S.H. Wong, The influence of surface chemistry on activated carbon adsorption of 2methylisoborneol from aqueous solution, Colloid Surface A: Physicochem. Eng. Aspects 179 (2001) 271-280.

[18] K.O. Nowack, F.S. Cannon, D.W. Mazyck, Enhancing activated carbon adsorption of 2-methylisoborneol: methane and steam treatments, Environ. Sci. Technol. 38 (2004) 276-284.

[19] G. Newcombe, J. Morrison, C. Hepplewhite, D.R.U. Knappe, Simultaneous adsorption of MIB and NOM onto activated carbon. II. Competitive effects, Carbon 40 (2002) 2147-2156.

[20] I.I. Salame, T.J. Bandosz, Study of water adsorption on activated carbons with different degrees of surface oxidation, J. Colloid Interf. Sci. 210 (1999) 367-374.

[21] A.P. Terzyk, The influence of activated carbon surface chemical composition on the adsorption of acetaminophen (paracetamol) in vitro. Part II. TG, FTIR, and XPS analysis of carbons and the temperature dependence of adsorption kinetics at the neutral $\mathrm{pH}$, Colloid Surface A: Physicochem. Eng. Aspects 177 (2001) 23-45.

[22] A. Swiatkowski, M. Pakula, S. Biniak, M. Walczyk, Influence of the surface chemistry of modified activated carbon on its electrochemical behavior in the presence of lead(II) ions, Carbon 42 (2004) 3057-3069.

[23] S. Biniak, G. Szymanski, J. Siedlewski, A. Swiatkowski, The characterization of activated carbons with oxygen and nitrogen surface groups, Carbon 35 (1997) 1799-1810.

[24] A. Derylo-Marczewska, A. Swiatkowski, B. Buczek, S. Biniak, Adsorption equilibria in the systems: aqueous solutions of organics-oxidized activated carbon samples obtained from different parts of granules, Fuel 85 (2006) 410-417.

[25] S.J. Randtke, V.L. Snoeyink, Evaluating GAC adsorption capacity, J. Am. Water Works Assoc. 75 (1983) 406

[26] G.A. Burlingame, I.H. Suffet, W.O. Pipes, Predominant bacterial genera in granular activated carbon water treatment systems, Can. J. Microbiol. 32 (1986) 226-230.

[27] N. Terauchi, T. Ohtani, K. Yamanaka, T. Tsuji, T. Sudou, K. Ito, Studies on a biological filter for musty odor removal in drinking-water treatment processes, Water Sci. Technol. 31 (1995) 229-235.

[28] M. Yagi, M. Kajino, U. Matsuo, K. Ashitani, T. Kita, T. Nakamura, Odor problems in Lake Biwa, Water Sci. Technol. 15 (1982) 311-321.

[29] C.Z. Liang, D.S. Wang, M. Yang, W. Sun, S.F. Zhang, Removal of earthy-musty odorants in drinking water by powdered activated carbon, J. Environ. Sci. Heal. A: Toxic/Hazard. Subst. Environ. Eng. 40 (2005) $767-$ 778.

[30] S.B. Watson, B. Brownlee, T. Satchwill, E.E. Hargesheimer, Quantitative analysis of trace levels of geosmin and MIB in source and drinking water using headspace SPME, Water Res. 34 (2000) 2818-2828.

[31] W. Heschel, E. Klose, On the suitability of agricultural by-products for the manufacture of granular activated carbon, Fuel 74 (1995) 17861791.

[32] B.K. Pradhan, N.K. Sandle, Effect of different oxidizing agent treatments on the surface properties of activated carbons, Carbon 37 (1999) $1323-1332$. 
[33] V. Boonamnuayvitaya, S. Sae-Ung, W. Tanthapanichakoon, Preparation of activated carbons from coffee residue for the adsorption of formaldehyde, Sep. Purif. Technol. 42 (2005) 159-168.

[34] A. Pakula, A. Swiatkowski, M. Walczyk, S. Biniak, Voltammetric and FT-IR studies of modified activated carbon systems with phenol, 4chlorophenol or 1,4-benzoquinone adsorbed from aqueous electrolyte solutions, Colloid Surface A: Physicochem. Eng. Aspects 260 (2005) $145-155$.
[35] J. Guo, A.C. Lua, Effect of surface chemistry on gas-phase adsorption by activated carbon prepared from oil-palm stone with pre-impregnation, Sep. Purif. Technol. 18 (2000) 47-55.

[36] K. Laszlo, E. Tombacz, K. Josepovits, Effect of activation on the surface chemistry of carbons from polymer precursors, Carbon 39 (2001) $1217-1228$. 